\title{
Time to move to PET-MR for cardiovascular imaging
}

\author{
Olivier Lairez, MD, PhD, ${ }^{\text {a }}$ Philip M. Robson, PhD, ${ }^{\mathrm{b}}$ and Zahi A. Fayad, $\mathrm{PhD}^{\mathrm{b}}$ \\ ${ }^{\text {a }}$ Cardiac Imaging Center, Toulouse University Hospital, Toulouse, France \\ ${ }^{b}$ Translational and Molecular Imaging Institute, Icahn School of Medicine at Mount Sinai, \\ New York
}

Received Jun 3, 2015; accepted Jun 3, 2015

doi: 10.1007/s12350-015-0206-4

\section{See related article, pp. 1102-1109}

In cardiovascular imaging, both positron emission tomography (PET) and magnetic resonance imaging (MRI) have emerged separately as essential components of evaluating disease. The high sensitivity of PET and its ability to target many biological processes such as cellular metabolism make PET well suited for cardiovascular perfusion and tissue viability assessment. In comparison, the subtle soft-tissue contrast, high temporal and spatial resolution, and sensitivity to perfusion contrast agents of MRI make it well suited for many cardiovascular applications, including morphological and functional cine imaging and imaging of scar tissue. The potential for similar complementary information in many other applications has lead to the development of hybrid PET-MR systems by all major vendors in either sequential or simultaneous configurations. There is now a clear motivation for hybrid PET-MR in cardiovascular imaging.

Despite the potential of hybrid systems, important technical issues must be addressed for PET-MR to become widely used in the clinic. ${ }^{1}$ Of primary concern is the required attenuation correction for PET imaging. In PET-CT imaging, the attenuation of x-rays in the CT image can be directly converted to attenuation values for the $511 \mathrm{keV}$ photons in PET imaging. In PET-MR imaging, the MRI-signal is proportional to proton density and not electron density as in CT imaging, and

\footnotetext{
Reprint requests: Zahi A. Fayad, PhD, Translational and Molecular Imaging Institute, Icahn School of Medicine at Mount Sinai, 1 Gustave L. Levy Place, New York 10029; zahi.fayad@mssm.edu J Nucl Cardiol 2016;23:1112-3.

$1071-3581 / \$ 34.00$

Copyright (C) 2015 American Society of Nuclear Cardiology.
}

therefore attenuation correction is a challenging proposition. Currently, an attenuation map is produced by image-based segmentation of an MRI image into four tissue classes (background air, lung, fat, and soft tissue), neglecting bone, ${ }^{2}$ followed by assignment of attenuation values measured separately.

Further issues for PET-MR include the attenuation of MRI receiver coils (both integrated body-transmit coils and spine coils embedded in the scan table, as well as flexible array-coils), the ability to estimate the attenuation map in large patients who exceed the useful field of view of the MRI system and the implementation of techniques to estimate activity in these areas (the MLAA technique referred to by Oldan et $\mathrm{al}^{3}$ ), and the availability of time-of-flight (TOF) PET imaging in hybrid systems (although by use of the silicon-based photo-multipliers in the latest generation of systems, TOF is becoming feasible in simultaneous hybrid PETMR systems).

These issues, as well as possible differences in the detectors, detector geometries, and reconstruction algorithms used in PET-CT and PET-MR systems make it challenging to compare directly the quantitative activity and SUV values measured by different systems.

In the current issue of the Journal, Oldan et al evaluated whether myocardial 18F-fluorodeoxyglucose (FDG)-PET-MR and PET-CT provide comparable images. ${ }^{3}$ The authors retrospectively analyzed myocardial FDG images of 27 patients undergoing PET-CT imaging followed by PET-MR imaging as part of a prior prospective study comparing the two modalities in oncology patients. They measured the mean standardized uptake value (SUV) for each of 17 standard segments and normalized the results to the brightest segment to give a relative intensity, as usually done in nuclear cardiology. The authors show that PET-MR values tend to be $10 \%$ higher than PET-CT values. This can be explained by the physiological increase of 
myocardial uptake over time ${ }^{4}$ (mean time interval between PET-CT and PET-MR was $42 \pm 13$ minutes). The overall correlation between PET-MR and PET-CT (with or without time-of-flight) raw and normalized values was good. Similar results were reported by others studies $^{5,6}$ and could also be attributed to the increasing myocardial glucose uptake with time. The important finding of Oldan et al is the absence of a significant difference between PET-CT and PET-MR modalities in the relative intensity values in myocardial segments. The results were similar when the analysis was done by gender and body mass index. As discussed by the authors, in nuclear cardiology, the relative intensity of the signal in an intense structure such as the heart is of primary concern. Significantly for use of PET-MR in clinical applications, these results suggest that polar map databases used for PET-CT could be applied to analysis of PET-MR studies. It is important to note that the relative PET-MR values (compared to PET-CT) tended to decrease as the PET-CT values increased and normalized values on PET-MR were greater than PET-CT values for overweight patients. These results suggest that values are comparable and homogeneous for standard ranges but diverge for extreme situations. The study by Oldan et al provides an initial response to the important question of comparability of data between PET-MR and PET-CT, notably, without including bone in the MRI-attenuation correction map. This study shows that results from PET-MR can be compared to results from PET-CT when relative intensity is of interest, encouraging future use of PET-MR in nuclear cardiology applications. Among the limitations of this study is the absence of validation of the results for patients with history of myocardial infarction, as well as the potential difference in absolute values of FDG uptake between both modalities, as in many other applications of PET-MR. Although another study suggests that myocardial SUVs from PET-CT and PET-MR are comparable, ${ }^{7}$ additional understanding of the differences in quantification by each system and validation in cardiovascular patients are still needed.

Currently, most of the indications of PET for cardiac imaging are for the assessment of myocardial viability or perfusion, which require relative myocardial uptake analysis. Oldan et al have demonstrated promising results that indicate PET-MR may be relevant for these applications, allowing us to also take advantage of the reduced radiation dose, and complementary information afforded by MRI over CT. But the recent advances and interest in hybrid scanners have also opened new perspectives for cardiac applications such as characterization of cardiac masses, study of inflammatory and infiltrative cardiomyopathies, and the evaluation of coronary atherosclerotic plaques. ${ }^{8,9}$ In addition, the need for serial follow-up and the development of new PET tracers will require absolute quantification of myocardial or coronary radiotracer uptake. Thus, there is a need to continue the development of PET-MR imaging methodology, building on promising early results, and to explore the exciting landscape ahead.

\section{References}

1. Kaufmann PA. Cardiac PET/MR: Big footprint-small step? J Nucl Cardiol 2015;22:225-6.

2. Hofmann M, Steinke F, Scheel V, Charpiat G, Farquhar J, Aschoff P, et al. MRI-based attenuation correction for PET/MRI: a novel approach combining pattern recognition and atlas registration. $\mathbf{J}$ Nucl Med 2008;49:1875-83.

3. Oldan J, Shah S, Brunken R, DiFilippo F, Obuchowski N, Bolen M. Do myocardial PET-MR and PET-CT FDG images provide comparable information? J Nucl Cardiol 2015. doi:10.1007/s12350015-0159-7.

4. Cheng G, Alavi A, Lim E, Werner TJ, Del Bello CV, Akers SR. Dynamic changes of FDG uptake and clearance in normal tissues. Mol Imaging Biol 2013;15:345-52.

5. Pace L, Nicolai E, Luongo A, Catalano OA, Soricelli A, Salvatore M. Comparison of whole-body PET/CT and PET/MRI in breast cancer patients: Lesion detection and quantitation of 18F-deoxyglucose uptake in lesions and in normal organ tissues. Eur J Radiol 2014;83:289-96.

6. Kershah S, Partovi S, Traughber BJ, Muzic RF Jr, Schluchter MD, O'Donnell JK, et al. Comparison of standardized uptake values in normal structures between PET/CT and PET/MRI in an oncology patient population. Mol Imaging Biol 2013;15:776-85.

7. Nensa F, Poeppel T, Tezgah E, Heusch P, Nassenstein K, Mahabadi AA, et al. Integrated FDG PET/MR imaging for the assessment of myocardial salvage in reperfused acute myocardial infarction. Radiology 2015;3:140564.

8. Ratib O, Nkoulou R. Potential applications of PET/MR imaging in cardiology. J Nucl Med 2014;55:40S-6S.

9. Nappi C, Altiero M, Imbriaco M, Nicolai E, Giudice CA, Aiello M, et al. First experience of simultaneous PET/MRI for the early detection of cardiac involvement in patients with Anderson-Fabry disease. Eur J Nucl Med Mol Imaging 2015;42:1025-31. 